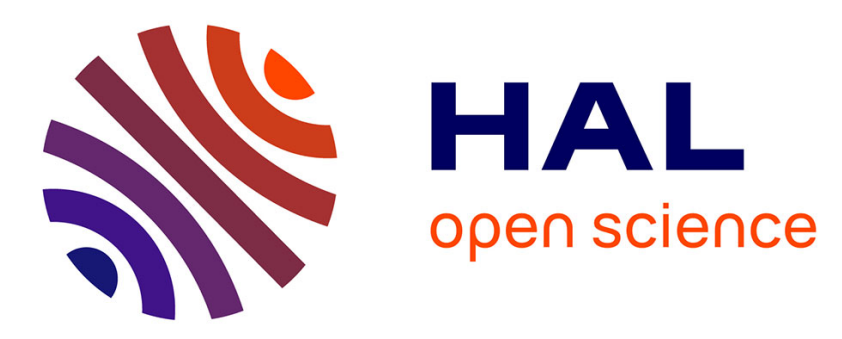

\title{
Probing slow dynamics of consolidated granular multicomposite materials by diffuse acoustic wave spectroscopy
}

Nicolas Tremblay, Éric Larose, Vincent Rossetto

\section{To cite this version:}

Nicolas Tremblay, Éric Larose, Vincent Rossetto. Probing slow dynamics of consolidated granular multicomposite materials by diffuse acoustic wave spectroscopy. Journal of the Acoustical Society of America, 2010, 127 (3), pp.1239-1243. 10.1121/1.3294553 . hal-02376188

\author{
HAL Id: hal-02376188 \\ https://hal.science/hal-02376188
}

Submitted on 1 Oct 2021

HAL is a multi-disciplinary open access archive for the deposit and dissemination of scientific research documents, whether they are published or not. The documents may come from teaching and research institutions in France or abroad, or from public or private research centers.
L'archive ouverte pluridisciplinaire HAL, est destinée au dépôt et à la diffusion de documents scientifiques de niveau recherche, publiés ou non, émanant des établissements d'enseignement et de recherche français ou étrangers, des laboratoires publics ou privés. 


\title{
Probing slow dynamics of consolidated granular multicomposite materials by diffuse acoustic wave spectroscopy
}

\author{
Nicolas Tremblay and Eric Larose ${ }^{\mathrm{a})}$ \\ Laboratoire de Géophysique Interne et Tectonophysique, CNRS and Université J. Fourier, \\ Grenoble BP 53 38041, France \\ Vincent Rossetto
Laboratoire de Physique et Modélisation des Milieux Condensés, CNRS and Université J. Fourier,
Grenoble BP 166 38042, France
}

(Received 2 September 2009; revised 14 December 2009; accepted 29 December 2009)

\begin{abstract}
The stiffness of a consolidated granular medium experiences a drop immediately after a moderate mechanical solicitation. Then the stiffness rises back toward its initial value, following a logarithmic time evolution called slow dynamics. In the literature, slow dynamics has been probed by macroscopic quantities averaged over the sample volume, for instance, by the resonant frequency of vibrational eigenmodes. This article presents a different approach based on diffuse acoustic wave spectroscopy, a technique that is directly sensitive to the details of the sample structure. The parameters of the dynamics are found to depend on the damage of the medium. Results confirm that slow dynamics is, at least in part, due to tiny structural rearrangements at the microscopic scale, such as inter-grain contacts. (C) 2010 Acoustical Society of America. [DOI: 10.1121/1.3294553]
\end{abstract}

PACS number(s): 43.25.Ts, 43.35.Yb, 43.40.Le, 43.35.Zc [RLW] Pages: 1239-1243

\section{INTRODUCTION: WHAT IS SLOW DYNAMICS?}

Depending on the magnitude of the solicitation applied to it, a consolidated granular medium, such as sedimentary rocks or concrete, can asymptotically react either in the elastic (reversible) regime, or in the brittle regime, where irreversible fractures develop. A few years ago, several articles from Guyer and co-workers and Ten Cate and co-workers (Ten Cate and Shankland, 1996; Guyer et al., 1998; Guyer and Johnson, 1999; Ten Cate et al., 2000) reported on an intermediate regime, where they observed non-linear elasticity. This regime is observed after imposing a strain of moderate amplitude $\left(10^{-6}\right)$ that does not generate any macroscopic damage. They called its time evolution as slow dynamics: it starts with a drop of the elastic modulus, followed by a logarithmic recovery of the sample stiffness toward its initial value after the strain is released. The $\log (t)$ recovery was found to be universal in granular solids of various composition (Ten Cate et al., 2000; Lacouture et al., 2003; Johnson and Sutin, 2005), humidity (Vakhnenko et al., 2004), or level of damage (Van Den Abeele et al., 2001). Most of the time, the evolution of the sample stiffness is monitored through the frequency of vibrational eigenmodes, using a technique named as non-linear elastic wave spectroscopy (Guyer and Johnson, 1999). More recently, Lobkis and Weaver (2009) proposed to monitor the stiffness of the material using the Larsen effect between two piezo-eletric transducers. The resonant frequency $f_{L}$ observed with a feedback loop was measured with a relative precision of the order $\delta f_{L} / f_{L} \approx 10^{-6}$, and was found to satisfyingly monitor the rigidity of the material. This latter technique offers simulta-

\footnotetext{
a) Author to whom correspondence should be addressed. Electronic mail: eric.larose@ujf-grenoble.fr
}

neously an increased sensitivity and a temporal resolution of a few ms, thus allowing to study slow dynamics over very short time-scales.

In this article, we develop an alternative method based on broadband diffuse ultrasound. We take advantage of coda waves that can be observed in multicomposite materials, where multiple scattering effects (characterized by the scattering mean free time $t^{\star}$ ) dominate absorption effects (characteristic time $\tau_{\text {abs }}$ ). Denoted by $T$, the central period of the ultrasound, and with $\Delta d$ as the time between two ultrasonic records, the time-scales in our experiment follow the hierarchy

$$
T<t^{\star}<\tau_{\text {abs }}<\Delta d .
$$

Similar to coda wave interferometry (CWI) (Poupinet et al., 1984; Roberts et al., 1992; Snieder et al., 2002) and to diffuse acoustic wave spectroscopy (DAWS) (Pine et al., 1988; Cowan et al., 2002), we study weak changes in the diffuse waveforms in amplitude and phase. We compare waveforms recorded after a moderate solicitation to those recorded before it. The solicitation consists in the impact of a small steel ball falling onto the sample from a controlled height $(1 \mathrm{~m}$ in general). The composition of the samples is displayed in Table I.

\section{EXPERIMENTAL SETUP}

In the first experiment, we use a cylindrical sample of 16 $\mathrm{cm}$ in diameter and $30 \mathrm{~cm}$ in height (sample 1). Two ultrasonic transducers are used: one as a source and one as a receiver (labeled as $S$ and $R$, respectively, in Fig. 1); they are $10 \mathrm{~cm}$ apart. Note that the lateral part of the transducer is inactive and rigid, so that surface (Rayleigh) waves are neither excited nor recorded. In this sample, the scattering mean 
TABLE I. Sample composition.

\begin{tabular}{llr}
\hline \hline Composition (in weight) & Weight & Density \\
\hline Cement: $17 \%$ & & \\
Fine sand: $31 \%$ & $12.5 \mathrm{~kg}$ & $2.2 \mathrm{~kg} / 1$ \\
Gravel: $43 \%$ & & \\
Water: $9 \%$ & & \\
\hline
\end{tabular}

free time is of the order of $12 \mu \mathrm{s}$, the transit time is of the order of $60 \mu \mathrm{s}$, and the absorption time is about $200 \mu \mathrm{s}$.

The lateral size of the active part of the transducers $(0.7$ $\mathrm{mm})$ is much smaller than the wavelength $(\lambda$ $=5 \mathrm{~mm}-5 \mathrm{~cm}$ at working frequencies), which makes them very sensitive to multiply scattered waves. The transducers are glued onto the sample using a hot chemical glue (phenylsalicylic acid) that solidifies with cooling (below $43{ }^{\circ} \mathrm{C}$ ). The ultrasonic experiment was performed several months after casting the samples. We send a $80 \mathrm{~V}$ broadband ultrasonic impulse with frequencies ranging from 50 to $500 \mathrm{kHz}$. The signal measured by the receiver is amplified, digitized, and stored in memory. A typical record $h_{d}(t)$ is shown in Fig. 2. The impulse response contains a hardly visible direct arrival, followed by a long lasting coda due to multiple scattered waves. The acquisition is repeated at various dates $d(d=0$ starts the experiment), over about $1 \mathrm{~h}$. At date $d_{I}$, we drop a small metallic ball $(m=30 \mathrm{~g})$ from a height $h=1.38 \mathrm{~m}$ onto the top face of the sample. The impact is about $15 \mathrm{~cm}$ from the source and receiver. The elastic energy released to the sample is $E_{\text {kin }}=m g h \approx 0.4 \mathrm{~J}$. Note that we observe no visible damage caused by this moderate impact.

\section{DATA PROCESSING}

From the set of ultrasonic data obtained during an experiment, we derive two quantities that depend on $d$. First, we measure the relative velocity change $\delta V_{d} / V$ within the material (Poupinet et al., 1984; Snieder et al., 2002; Lobkis and Weaver, 2003). Second, we measure the remnant decorrelation $K_{d}$ of the waveforms after correcting for the effect of the relative velocity change (Lobkis and Weaver, 2003).

\section{A. Relative velocity change $\delta V / V(C W I)$}

Let us describe the two steps of our data processing in details. To measure the relative velocity change, we compare the phase of the waveforms acquired at the date $d$ to the phase of the initial record $(d=0)$. Following SensSchönfelder and Wegler (2006), we interpolate and resample

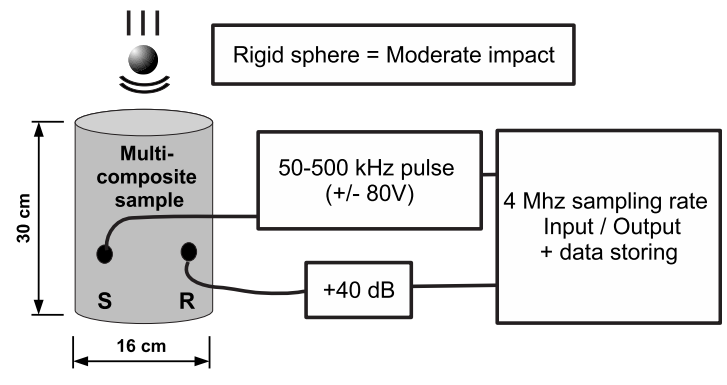

FIG. 1. Schematic view of the experimental setup for sample 1.

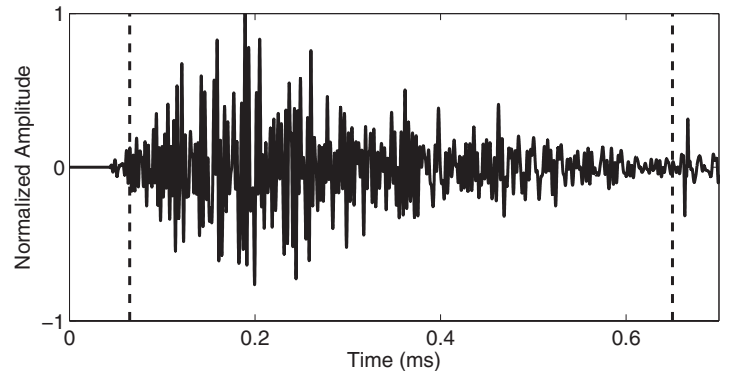

FIG. 2. Typical record collected through a concrete sample. The integration time-window is marked by the two dashed vertical lines at 0.05 and $0.65 \mathrm{~ms}$. The initial impulse duration is about $10 \mu \mathrm{s}$.

$h_{d}(t)$ at times $[1+\epsilon] t$; this corresponds to stretching the time axis of the record by a factor of $1+\epsilon$. Then we evaluate the correlation coefficient between $h_{0}$ and $h_{d}$

$$
X_{d}(\epsilon)=\frac{\int h_{0}(t) h_{d}([1+\epsilon] t) d t}{\sqrt{\int h_{0}^{2}(t) d t} \sqrt{\int h_{d}^{2}([1+\epsilon] t) d t}},
$$

where the integration is performed over a time-window larger than the period $T$. This time-window is marked by two vertical broken lines in Fig. 2. This calculation is repeated for various values of the parameter $\epsilon$. The parameter $\epsilon$ maximizing $X_{d}$ corresponds to the actual relative velocity change

$$
\epsilon_{\max }=-\delta V_{d} / V \text {. }
$$

The evolution of $\delta V_{d} / V$ as a function of $d$ is displayed in Fig. 3 (top). Note that the ball impact occurs at date $d_{I}$ $=270 \mathrm{~s}$ after starting the experiment. Before the impact, there is no relative velocity change, indicating that the medium is at rest. Immediately after the impact, the velocity is noticeably decreased. Since the density of the material is not changed, and since diffuse waves are mainly in the trans-
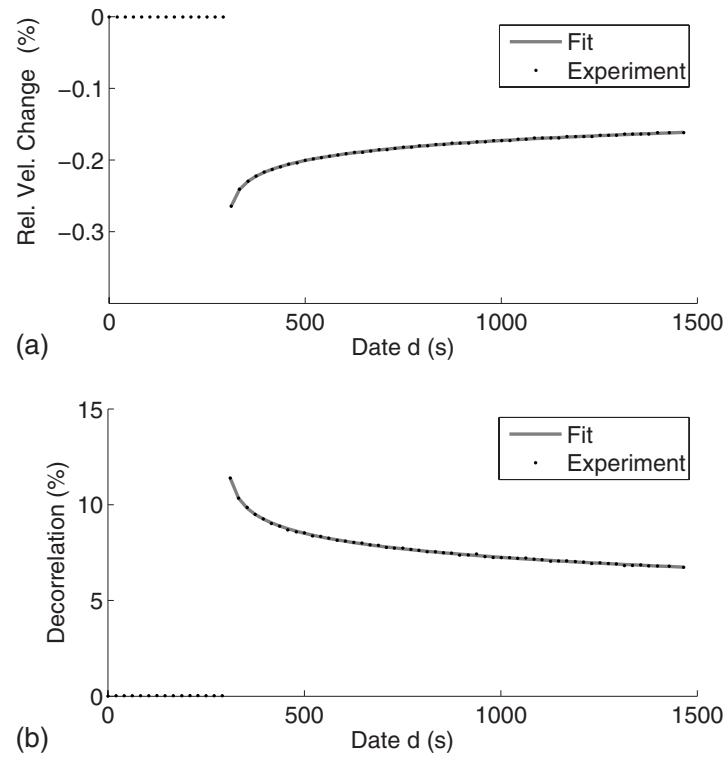

FIG. 3. Relative velocity change $\delta V / V$ (top) and decorrelation (bottom) versus the date $d$ of the measurement. Impact is at $d_{I}=270 \mathrm{~s}$. Thermal effects are negligible. A logarithmic fit is added to each experimental curve. 
verse (shear) polarization, this velocity drop is principally due to a drop of rigidity of the material. Then, the material stiffness rises back toward its initial value.

\section{B. Remnant decorrelation K (DAWS)}

In a second step, we use the dilated records to evaluate the remnant decorrelation coefficient $K_{d}$ between the waveforms, which simply reads (Pine et al., 1988; Cowan et al., 2002)

$$
K_{d}=1-X_{d}\left(\epsilon_{\max }\right) .
$$

The time evolution of $K$ as a function of $d$ is plotted in Fig. 3 (bottom). Again, the record is very stable (no change) before the impact. After the impact, the ultrasonic waveform differs significantly from the reference, then the decorrelation from the reference waveform decreases over several hundreds of seconds. The decorrelation $K$ defined in Eq. (4) cannot be solely associated to a difference of relative velocity changes for different waves (for instance, between compressional and shear waves), contrary to the decorrelation studied by Lobkis and Weaver (2003). Also, the decorrelation cannot be attributed to changes in the bulk of the grains, which would require much more energy than the one released by the ball. Here, it corresponds to a feeble change in the scattering properties of the concrete sample, for instance, a small rearrangement of contacts between grains.

It is important to note that thermal changes are also well known to affect the average velocity within the sample (Lobkis and Weaver, 2003; Snieder et al., 2002; Larose et al., 2006), and consequently, the decorrelation. Relative velocity changes due to temperature variations are found to be negligible in our experiment, as can be noticed by the flat curve in Fig. 3, from $t=0$ to $t=270 \mathrm{~s}$. The decorrelation of the waveforms before the impact is actually near $0.1 \%$, and is attributed to the noise generated by the electronic devices. We neglect it in the following. Note also that the changes in intensity of the diffuse waveforms (say, the quality factor of the coda) was observed to show some recovery (Ten Cate $e t$ al., 2000), but this effect is not studied here and is beyond the scope of the present paper.

\section{THE PHYSICAL PARAMETERS OF SLOW DYNAMICS}

The time evolutions of both $\delta V / V$ and $K$ fit logarithmic laws (with $d$ and $d_{I}$ in seconds)

$$
\begin{aligned}
& \delta V_{d} / V=A^{\prime}-m^{\prime} \log _{10}\left(d-d_{I}\right), \\
& K_{d}=A-m \log _{10}\left(d-d_{I}\right) .
\end{aligned}
$$

Such a logarithmic evolution, valid if $d \ll 10^{A / m}$, is referred to as slow dynamics. $A$ and $m$ are the fit parameters, where $A$ is the extrapolated decorrelation $1 \mathrm{~s}$ after the impact, and $m$ is the slope of the logarithmic decay. $10^{A / m}$ represents the recovery time (in seconds), which is the time the system needs to recover its initial state. In general, this recovery time is of the order of $10^{4}-10^{5} \mathrm{~s}$. Parameters $A$ and $m$ were found to depend on the experimental apparatus, but Eq. (6) was found to describe the dynamics in all cases. Ten Cate et al. (2000)

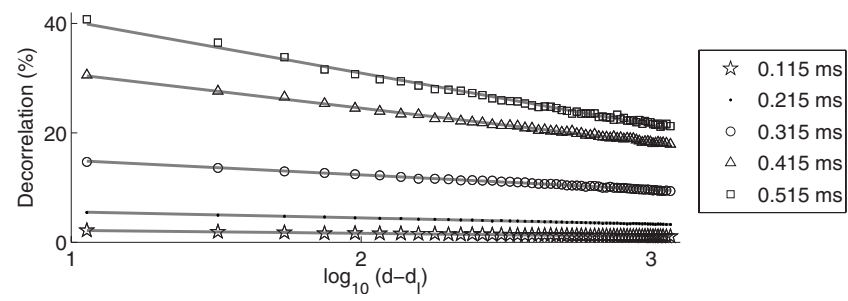

FIG. 4. Decorrelation versus the date of the measurement (in log scale) for various time-windows in the coda. The central time of each window is displayed in the box. The recovery time $10^{A / m}$ is found to be constant in the whole record.

mentioned a break away from the log recovery after $1000 \mathrm{~s}$. In our experiments, we should certainly expect an erosion of the log dynamics at some time. In particular, we must consider the possibility that part of the change in our experiments might be irreversible, and that the recovery might saturate. This could be ascribed to irreversible (but invisible) damages caused by the impact. As we could not monitor the sample longer than $1000 \mathrm{~s}$, we cannot address this issue properly.

\section{A. Slow dynamics versus time in the coda}

Let us now study the parameters $A$ and $m$ in more detail. As a first test, we evaluate these parameters at different times $t$ in the coda. To that end, we split the initial time window (see Fig. 2) into five consecutive time-windows with length of $0.12 \mathrm{~ms}$. The decorrelation $K_{d}$ for these five windows is displayed in Fig. 4. $A$ and $m$ were found to increase with the time in the coda, confirming that late diffuse waves are much more sensitive to weak perturbation than early arrivals. Interestingly enough, the recovery time $10^{A / m}$ is constant within the whole record, which confirms that all timewindows (thus all wave paths) undergo the same slow dynamics phenomenon.

\section{B. Slow dynamics versus frequency}

To study the frequency dependence of slow dynamics, we filter the whole record into different frequency bands; we observe that $A / m$ is constant in the $50-500 \mathrm{kHz}$ frequency range. That the same dynamics was observed at different wavelength might suggest that there is no characteristic length in the rearrangement of the material structure. This confirms the assumption commonly made by previous authors (Ten Cate et al., 2000) that the changes occur at various scales, following the probable continuous distribution of energy barriers.

\section{Spatial dependence of slow dynamics}

In the initial works on slow dynamics (Ten Cate and Shankland, 1996; Guyer et al., 1998), the medium was subject to a global excitation, and resulted in a global change in the sample stiffness. The excitation presented here is a local impact (as in (Lobkis and Weaver, 2009). Determining whether the change induced by the ball impact is global (induced by the shock wave), or local is of some importance. To that end, we deploy a linear array of four sensors $10 \mathrm{~cm}$ 


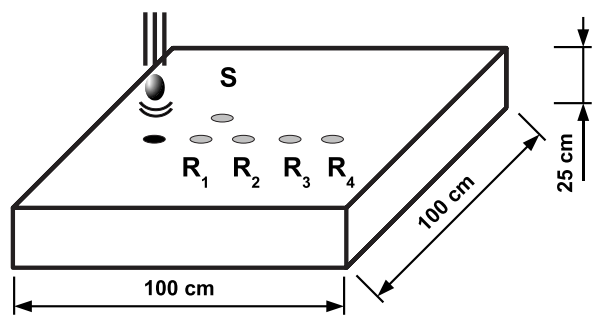

FIG. 5. Experimental setup for sample 2. Four receivers $\left(R_{1-4}\right)$ are aligned with the impact location. The source $S$ is $10 \mathrm{~cm}$ off the impact-receiver axis.

away from the impact location and from the source transducer. Because the initial sample is not large enough, we use a different sample (2) of similar composition but whose dimensions are now $1 \times 1 \times 0.25 \mathrm{~m}^{3}$ (see Fig. 5). The array has a spatial pitch of about $10 \mathrm{~cm}$. The decorrelation $K$ is found to strongly depend on the impact-receiver distance (Fig. 6). We therefore confirm the assumption issued by Lobkis and Weaver (2009) that the changes induced by the ball impact are located in the vicinity of the impact, and the seismic (shock) wave emitted by the impact has negligible effect on the material stiffness at large distances.

\section{TENTATIVE APPLICATION TO ON-SITE DAMAGE ESTIMATION}

Last, we address the issue of damage estimation through DAWS. Consider two blocks of concrete of similar constitution (see Table I) and of the same geometry (see sample 1); the first one is intact and the other one is damaged after undergoing a $30 \mathrm{MPa}$ load. Both samples are tested using the same experimental protocol, and data are processed in the same time window and frequency range. Resulting decorrelations $K_{d}$ are plotted in Fig. 7. Similar to Van Den Abeele et al. (2001), we observe that damage increases considerably the amplitude of change in the material, and thus, the absolute decorrelation $A$ together with the speed of recovery $m$. Provided that the experimental protocol is rigorously similar from one site to another, this latter result suggests a possible route for on-site concrete damage assessment.

\section{CONCLUSION}

In this article, we studied the slow dynamics behavior of concrete after a ball impact of moderate energy. We observed

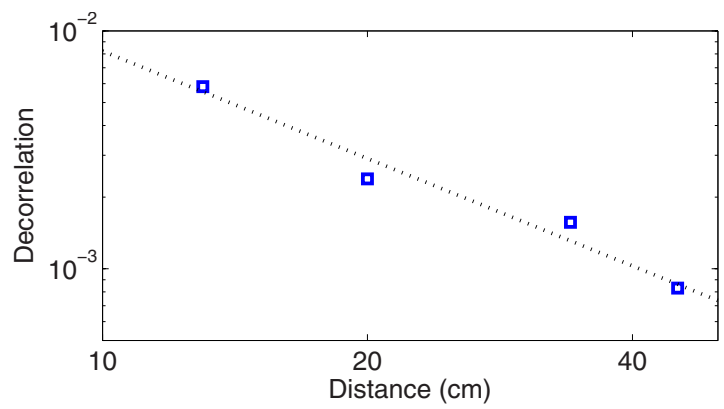

FIG. 6. (Color online) Decorrelation of ultrasonic waveforms obtained a few ms right after the impact in log-log scale, at different distances from the impact. Squares represent the experimental data, while dots represent a power law with the exponent -1.5 .

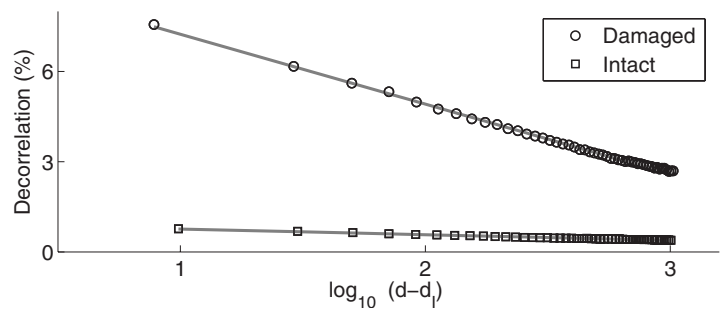

FIG. 7. Slow dynamics observed in undamaged and damaged concretes.

a drop of the acoustic velocity (probed by CWI, Poupinet $e t$ al., 1984) and a decorrelation of the diffuse waveforms (probed by DAWS). Then the material recovers over characteristic times ranging from 100 to several $1000 \mathrm{~s}$. In our experiments, the $\log (t)$ recovery was observed at all time in the coda, and at different frequencies; this does not allow to exhibit any characteristic length, time, or energy. This feature is in agreement with a fractal structure of concrete. Slow dynamics is thus interpreted as a series of jumps of energy barriers having a continuous distribution. In principle, slow dynamics could be either due to a homogeneous change in mechanical characteristics of all constituents, or to local rearrangements of inter-grain contacts. As we observe a noticeable decorrelation of the diffuse waveforms that cannot be solely ascribed to a homogeneous change in the material stiffness, we defend the second interpretation; slow dynamics is due to a collection of local changes in inter-grain contacts, which simultaneously result in changes in scattering properties and in a decrease in the effective wave velocity. The rearrangements of inter-grain contacts in our experiments are somehow analogous to those observed in solid friction (Lomnitz, 1962; Pandit and Savage, 1973; Karner and Marone, 1998). As concrete is an example of consolidated multicomposite granular material, we also believe that our laboratory experiment shares analogy with the time evolution of shallow sedimentary geomaterials after an earthquake (Sawazaki et al., 2006; Brenguier et al., 2008). In our experiments, slow dynamics parameters $A$ and $m$ where found to be very sensitive to experimental apparatus, particularly to the impact energy and position. While keeping these features perfectly constant and the experiment perfectly reproducible, we observed that the slow dynamics parameters were also found to strongly depend on damage, which suggest that they could characterize the aging of concrete structures. Since the experimental apparatus is light and easy to handle, it could have some practical interest in structural health monitoring, especially in civil engineering, or to perform down hole geomaterial characterization, provided that a reproducible and controlled impact could be delivered to the medium at depth.

\section{ACKNOWLEDGMENTS}

The authors would like to thank R. L. Weaver and P. A. Johnson for attracting their attention on slow dynamics. This work was funded by the ANR SISDIF Grant No. 08-JCJC0066.

Brenguier, F., Campillo, M., Hadziioannou, C., Shapiro, N. M., Nadeau, R. M., and Larose, E. (2008). "Postseismic relaxation along the San Andreas fault at Parkfield from continuous seismological observations," Science 
321, 1478-1481.

Cowan, M. L., Jones, I. P., Page, J. H., and Weitz, D. A. (2002). "Diffusing acoustic wave spectroscopy," Phys. Rev. E 65, 066605.

Guyer, R. A., and Johnson, P. A. (1999). "Non-linear mesoscopic elasticity: Evidence for a new class of materials," Phys. Today 52(4), 30-36.

Guyer, R. A., McCall, K. R., and Abeele, K. V. D. (1998). "Slow elastic dynamics in a resonant bar of rock," Geophys. Res. Lett. 25, 1585-1588.

Johnson, P., and Sutin, A. (2005). "Slow dynamics and anomalous fast dynamics in diverse solids," J. Acoust. Soc. Am. 117, 124-130.

Karner, S. L., and Marone, C. (1998). "The effect of shear load healing in simulated fault gouge," Geophys. Res. Lett. 25, 4561-4564.

Lacouture, J., Johnson, P., and Cohen-Tenoudji, F. (2003). "Study of critical behavior in concrete during curing by application of dynamic linear and nonlinear means," J. Acoust. Soc. Am. 113, 1325-1332.

Larose, E., de Rosny, J., Margerin, L., Anache, D., Gouedard, P., Campillo, M., and VanTiggelen, B. (2006). "Observation of multiple scattering of $\mathrm{kHz}$ vibrations in a concrete structure and application to weak changes monitoring," Phys. Rev. E 73, 016609.

Lobkis, O. I., and Weaver, R. L. (2003). "Coda-wave interferometry in finite solids: Recovery of P-to-S conversion rates in an elastodynamic billiard," Phys. Rev. Lett. 90, 254302.

Lobkis, O. I., and Weaver, R. L. (2009). "On the Larsen effect to monitor small fast changes in materials," J. Acoust. Soc. Am. 125, 1894-1905.

Lomnitz, C. (1962). "Application of the logarithmic creep low to stress wave attenuation in the solid earth," J. Geophys. Res. 67, 365-368.

Pandit, B. I., and Savage, J. C. (1973). "An experimental test of Lomnitz's theory of internal friction in rocks,” J. Geophys. Res. 78, 6097-6099.

Pine, D. J., Weitz, D. A., Chaikin, P. M., and Herbolzheimer, E. (1988).
"Diffusing-wave spectroscopy," Phys. Rev. Lett. 60, 1134-1137.

Poupinet, G., Ellsworth, W. L., and Frechet, J. (1984). "Monitoring velocity variations in the crust using earthquake doublets: An application to the Calaveras fault, California,” J. Geophys. Res. 89, 5719-5731.

Roberts, P. M., Phillips, W. S., and Fehler, M. (1992). "Development of the active doublet method for measuring small velocity and attenuation changes in solids," J. Acoust. Soc. Am. 91, 3291-3302.

Sawazaki, K., Sato, H., Nakahara, H., and Nishimura, T. (2006). "Temporal change in site response caused by earthquake strong motion as revealed from coda spectral ratio measurement," Geophys. Res. Lett. 33, L21303.

Sens-Schönfelder, C., and Wegler, U. C. (2006). "Passive image interferometry and seasonal variations of seismic velocities at Merapi volcano, Indonesia," Geophys. Res. Lett. 33, L21302.

Snieder, R., Grêt, A., Douma, H., and Scales, J. (2002). "Coda wave interferometry for estimating nonlinear behavior in seismic velocity," Science 295, 2253-2255.

Ten Cate, J. A., and Shankland, T. J. (1996). "Slow dynamics in the nonlinear elastic response of Berea sandstone," Geophys. Res. Lett. 23, 30193022 .

Ten Cate, J. A., Smith, E., and Guyer, R. A. (2000). "Universal slow dynamics in granular solids," Phys. Rev. Lett. 85, 1020-1023.

Vakhnenko, O. O., Vakhnenko, V. O., Shankland, T. J., and TenCate, J. A. (2004). "Strain induced kinetics of inter-grain defects as the mechanism of slow dynamics in the nonlinear resonant response of humid sandstone samples," Phys. Rev. E 70, 015602.

Van Den Abeele, K., Sutin, A., Carmeliet, J., and Johnson, P. (2001). "Micro-damage diagnostics using nonlinear elastic wave spectroscopy (NEWS)," NDT Int. 34, 239-248. 